\title{
Nachruf
}

\section{Prinzip Erfahrung. Helmut Schelsky zum Gedächtnis}

\section{Hermann Lübbe}

Universität Zürich, Philosophisches Seminar, Rämistraße 71, CH-8006 Zürich

Wir kennen die Philosophie in ihrer Zuständigkeit für das, was unabhängig von aller Erfahrung gilt, wie die Regeln der Logik zum Beispiel oder die universalen Grundsätze der Moral. Zugleich gibt es die Philosophie, die den Hunger nach Empirie weckt und verstärkt. Die Gesellschaftsphilosophie des sogenannten Deutschen Idealismus ist dafür ein herausragendes Beispiel. Helmut Schelsky hat diese Philosophie in ihren Voraussetzungen und Folgen von Hobbes über Kant und Fichte bis zu Marx als Student Anfang der dreißiger Jahre in ihrer Leipziger Brechung im Umkreis Hans Freyers und Arnold Gehlens kennengelernt. Ihnen beiden ist er auch nach dem Kriege in Freundschaft verbunden geblieben.

Entschiedener als sie vollzog er die fachliche Wendung zur Soziologie - zunächst als Professor an der gewerkschaftsnahen Akademie für Gemeinwirtschaft in Hamburg sowie an der Universität daselbst. Seine familien- und jugendsoziologischen Forschungen haben ihn in den fünfziger Jahren rasch bekannt, ja berühmt gemacht. Die Fakten waren darin mit der Philosophie des Interesses, das diese Fakten uns suchen läßt, überzeugend verbunden. Das ist es, was diese Soziologie weit über den Kreis der Fachwissenschaftler hinaus wirksam gemacht hat. Es bewegte, zum Beispiel, das Publikum, als Resultat soziologischer Analyse bestätigt $\mathrm{zu}$ erhalten, was schon die Lebenserfahrung $\mathrm{zu}$ lehren schien, daß nämlich in Zeiten gesellschaftlicher Umbrüche die soziale Bedeutung der Familienbindung zunimmt. - Analog breitenwirksam waren auch Schelskys bildungssoziologische Thesen. Nicht wenige Pädagogen zeigten sich von Schelskys Nachweis betroffen, daß Schulen und Hochschulen heute die wichtigsten Institutionen für die Verteilung von Sozialchancen sind. Aber eben das ist es doch, was heute über alle sozialen Schichtdifferenzen hinweg Eltern wie nie zuvor um den Schulerfolg ihrer Kinder besorgt sein läßt. Die Geschwindigkeit in der Expansion unserer Bildungseinrichtungen, wie sie in jüngstvergangenen
Jahren stattgefunden hat, entspricht dem. - Vor wenigen Jahren noch galt Empörung über sexuelle Freizügigkeit auf Filmleinwänden als lächerlich. Inzwischen hat man feministische Anschläge auf Sexshops erlebt. Helmut Schelskys „Soziologie der Sexualität" (1955), die ein Bestseller wurde, hätte dergleichen vorauszusagen erlaubt.

Schelskys soziologische Studien waren aktuell, indem sie den Zeitgeist, anstatt sich ihm anzupassen, verständlich machten. $\mathrm{Zu}$ den Bedingungen ihrer außerordentlichen Wirkung gehört überdies Schelskys große Mitteilungs- und Darstellungskunst. Sein Titel "Die skeptische Generation" (1957), zum Beispiel, erwies sich als schlagwortreif.

Es ist wahr: Die akademische Jugendbewegung der späten sechziger Jahre, die in den Ereignissen des Pariser Mai 1968 auf ihren Höhepunkt kam, hat diesen Titel dann in wichtigen Hinsichten falsifiziert. Nicht Skepsis war ja die dominante Anmutungsqualität dieser neuen Jugendbewegung, vielmehr - zumal in Deutschland - neue ideologische Gläubigkeit, die in Extremfällen Züge eines neuen intellektuellen Fanatismus annahm. Schelsky selbst hat vorausgesehen, daß es bei der generationsspezifischen "Skepsis“ der Jugend der fünfziger Jahre nicht bleiben werde. „Ich erwarte eine ,sezessionistische“ Jugendgeneration" - so schrieb er im Schlußwort seines Buches von 1957.

Gleichwohl: Schelskys Sympathie scheint der „skeptischen Generation“ gehört zu haben. Seine Schilderung dieser Generation enthält Elemente normativer Auszeichnung ihres Verhaltens. Die skeptische Generation war, sozusagen, eine modernisierungsbereite Generation. Aus dieser Perspektive gesehen waren die Ideologie-Renaissancen der später sechziger und frühen siebziger Jahre, die den historisch-politischen Umständen entsprechend sich vor allem spätmarxistisch orientierten, weniger Bewegungen neuen Engagements bei der Wirklichkeit als Bewegungen der Flucht aus ihr. 
Helmut Schelsky war ein Anti-Ideologe, das heißt ein Mann lebenserfahrungsbegründeter Skepsis gegen Ansprüche von Großtheorien, die auf $\mathrm{Zu}-$ kunftsgesellschaften spezialisiert sind und so alles besser als jene Bürgermehrheiten wissen, die mit Überkommenem gute Erfahrungen gemacht haben und es daher fortschreiben möchten. In seinem berühmten kleinen Buch „Der Mensch in der wissenschaftlichen Zivilisation" (1961) hat Schelsky so die Industriegesellschaft verteidigt und den Zwang der Sachgesetzlichkeiten, die in ihr wirksam sind, als Entlastungsvorgang analysiert. Man hat darin eine "technokratische“ Verweigerung der Anerkennung moralischer und politischer Geltungsansprüche erkennen wollen. In Wahrheit ist, selbstverständlich, auch für Schelsky die Entwicklung der Industriegesellschaft nicht ein moralisch erblindeter Selbstlauf. Vielmehr bleibt es Sache des Gemeinsinns, diese Entwicklung nach Nutzen und Nachteil zu validieren. Anerkennung von Sachgesetzlichkeiten ruiniert nicht den Bürgersinn, sondern erhöht die Effizienz von Planung und Steuerung.

Das ist es, wovon auch der Universitätsplaner Helmut Schelsky sich leiten ließ. Damit begann er nach seinem Wechsel an die Universität Münster 1960. Sein Buch ,Einsamkeit und Freiheit" (1963) ist nicht der Entwurf einer technokratischen Kaderschmiede, vielmehr, auf den Spuren Wilhelm von Humboldts, der Versuch, unter Bedingungen der Massenakademisierung die Universität als Stätte erstrangiger wissenschaftlicher Forschung zu erhalten und zugleich den Bornierungen des Spezialistentums entgegenzuwirken. Die Universität Bielefeld wurde von Helmut Schelsky in dieser Absicht geplant. Einiges davon hat sich als produktiv erwiesen; anderes ist deutschen akademischen Sonderentwicklungen zum Opfer gefallen.
Daß Wissenschaft und akademische Bildung den Gemeinsinn nicht stiften, sondern zur kulturellen Voraussetzung haben - davon geht auch Schelskys Intellektuellen-Kritik aus. Politische und moralische Urteilskraft wird nicht seminarintern aus dem Geiste der Soziologie geboren, und auch in den Medien hat sie nicht ihren Ursprungsort. Ihre kulturelle Basis sind vielmehr traditional bewährte Lebenserfahrung aus Tätigkeiten in privater, beruflicher und politischer Verantwortung für Personen und Sachen. In diesem Sinn hat Helmut Schelsky das „Prinzip Erfahrung“ gegen Blochs "Prinzip Hoffnung" aufgeboten.

Schelskys Stilisierung der Intellektuellen zu einer neuen, kämpfenden Klasse (,Die Arbeit tun die anderen“, 1975) war eine Übertreibung in polemischer Absicht. Ihr sachliches Recht hat sie in gewissen Folgen der Tatsache, daß in einer medial integrierten Gesellschaft der Horizont unserer Informiertheit zwangsläufig rascher wächst als die Reichweite unserer Urteilskraft. Die Ideologieanfälligkeit der Intellektuellen, an die sich Schelsky sehr wohl auch aus seiner eigenen Studentenzeit zu erinnern wußte, wurzelt nicht zuletzt in dieser Inkongruenz, und wie vor ihm andere LiberalKonservative, Raymond Aron zum Beispiel, hat er sie kritisiert. Wie diesen hat man ihn einen Konservativen genannt, und er war es wie viele andere Liberale inzwischen auch: als Verteidiger liberaler Kultur und Lebensordnung gegen die Gebildeten unter ihren neuen Verächtern.

Nicht leicht verständlich bleibt die Resignation, die Helmut Schelsky in seinen letzten Lebensjahren in einen Winkel des Burgenlandes sich zurückziehen ließ, während er doch die Wirkung und Anerkennung seines Lebenswerkes, die weltweit waren, hätte genießen sollen. 